\title{
Uso de plantas medicinais pelos professores, estudantes e seus familiares durante a pandemia da COVID-19: um relato de experiência
}

\author{
Use of medicinal plants by teachers, students and their families during the COVID-19 pandemic:
} an experience report

Uso de plantas medicinales por profesores, estudiantes y sus familias durante la pandemia COVID19: un informe de experiencia

Eliane Alves Lustosa ORCID: https://orcid.org/0000-0002-8264-2204 Universidade Federal de Campina Grande, Brasil E-mail: elianelustosa18@hotmail.com

Emmanuelle de Kássia Nóbrega ORCID: https://orcid.org/0000-0002-8727-7488

Universidade Federal de Campina Grande E-mail: manukassia83@gmail.com Lucas de Brito Silva ORCID: https://orcid.org/0000-0002-7852-8011 Universidade Federal de Campina Grande E-mail: lucasbrito.debs@gmail.com

Maria Cecília Pereira Marinho ORCID: https://orcid.org/0000-0001-9030-2907

Universidade Federal de Campina Grande E-mail: cecilia.marinho@estudante.ufcg.edu.br Ozanna Soares Medeiros de Araújo ORCID: https://orcid.org/0000-0003-0951-7295

Universidade Federal de Campina Grande E-mail: ozanna.medeiros55@gmail

Yasmim Radija de Andrade Alves ORCID: https://orcid.org/0000-0003-2470-0426

Universidade Federal de Campina Grande

E-mail: yasmim.radija@estudante.ufcg.edu.br Darly Silva de Araújo ORCID: https://orcid.org/0000-0002-4074-6398 Universidade Federal de Campina Grande E-mail:darlydaraujo@gmail.com

Aleson Pereira de Sousa

ORCID: https://orcid.org/0000-0002-3430-477X Universidade Federal da Paraíba, Brasil

E-mail: aleson_155@hotmail.com

Luciano de Brito Junior

ORCID: https://orcid.org/0000-0002-1787-4316 Universidade Federal de Campina Grande

E-mail: luciano.brito@professor.ufcg.edu.br

Abrahão Alves de Oliveira Filho ORCID: https://orcid.org/0000-0002-7466-9933 Universidade Federal de Campina Grande E-mail: abrahao.farm@gmail.com

Maria das Graças Veloso Marinho de Almeida ORCID: https://orcid.org/0000-0002-8834-2077 Universidade Federal de Campina Grande E-mail:mgvmarinho1@gmail.com

\section{Resumo}

Este artigo tem como finalidade relatar a experiência de um projeto de extensão realizado por professores e estudantes da Universidade Federal de Campina Grande em uma comunidade escolar no município de São Mamede, Paraíba. O projeto teve como objetivo sensibilizar a comunidade sobre o uso de plantas medicinais utilizadas no controle de infecções respiratórias agudas durante a pandemia da COVID-19. O público alvo foram professores, estudantes e seus familiares da Escola Cidadã Integral Técnica Seráfico Nóbrega, no município de São Mamede/PB. Inicialmente, houve a realização de reuniões síncronas com os participantes e orientadores para o planejamento e elaboração das 
atividades que seriam desenvolvidas durante o período de vigência do projeto. Posteriormente, foi realizada pelos alunos extensionistas uma pesquisa com base na literatura acerca das plantas medicinais, sendo selecionadas dez plantas capazes de auxiliar no tratamento ou prevenção das infecções respiratórias agudas. Após, foram elaborados materiais como panfletos, textos e vídeos a respeito do tema, sendo postados na plataforma Google Classroom ${ }^{\circledR}$, e foram realizadas palestras, por meio da plataforma Google Meet@. Também foi desenvolvido um perfil nas redes sociais (Instagram $\left.{ }^{\circledR}\right)$ que permitiu maior alcance das informações. Diante disso, o projeto teve êxito em suas condutas já que o público alvo foi instruído e participativo nas atividades, o que possibilitou a assimilação de conhecimentos em relação à utilização das plantas medicinais, unindo o conhecimento popular e o científico.

Palavras-chave: Infecções respiratórias agudas; Plantas medicinais; Fitoterapia; COVID-19.

\begin{abstract}
This article aims to report the experience of an extension project carried out by teachers and students at the Federal University of Campina Grande in a school community in the city of São Mamede, Paraíba. The project aimed to raise community awareness about the use of medicinal plants used to control acute respiratory infections during the COVID-19 pandemic. The target audience were teachers, students and their families from Escola Cidadã Integral Técnica Serafico Nóbrega, in the city of São Mamede/PB. Initially, there were synchronous meetings with participants and supervisors for the planning and elaboration of activities that would be developed during the period of validity of the project. Subsequently, extension workers carried out a research based on the literature on medicinal plants, with ten plants capable of helping in the treatment or prevention of acute respiratory infections being selected. Afterwards, materials such as pamphlets, texts and videos about the theme were prepared, being posted on the Google Classroom ${ }^{\circledR}$ platform, and lectures were given through the Google Meet ${ }^{\circledR}$ platform. A profile on social networks (Instagram $\left.{ }^{\circledR}\right)$ was also developed, which allowed for a greater reach of information. Therefore, the project was successful in its conduct since the target audience was educated and participated in the activities, which enabled the assimilation of knowledge in relation to the use of medicinal plants, uniting popular and scientific knowledge.
\end{abstract}

Keywords: Acute respiratory infections; Medicinal plants; Phytotherapy, COVID-19.

\title{
Resumen
}

Este artículo tiene como objetivo reportar la experiencia de un proyecto de extensión realizado por docentes y estudiantes de la Universidad Federal de Campina Grande en una comunidad escolar de la ciudad de São Mamede, Paraíba. El proyecto tenía como objetivo sensibilizar a la comunidad sobre el uso de plantas medicinales utilizadas para controlar las infecciones respiratorias agudas durante la pandemia de COVID-19. El público objetivo fueron profesores, estudiantes y sus familias de la Escola Cidadã Integral Técnica Serafico Nóbrega, en la ciudad de São Mamede / PB. Inicialmente, se realizaron reuniones sincrónicas con participantes y supervisores para la planificación y elaboración de las actividades que se desarrollarían durante el período de vigencia del proyecto. Posteriormente, los extensionistas realizaron una investigación basada en la literatura sobre plantas medicinales, seleccionándose diez plantas capaces de ayudar en el tratamiento o prevención de infecciones respiratorias agudas. Posteriormente, se prepararon materiales como folletos, textos y videos sobre el tema, que se publicaron en la plataforma Google Classroom ${ }^{\circledR}$, y se dictaron conferencias a través de la plataforma Google Meet®. También se desarrolló un perfil en redes sociales (Instagram $\left.{ }^{\circledR}\right)$, que permitió un mayor alcance de la información. Por lo tanto, el proyecto tuvo éxito en su conducción ya que el público objetivo fue educado y participó de las actividades, lo que permitió la asimilación de conocimientos en relación al uso de plantas medicinales, uniendo el conocimiento popular y científico.

Palabras clave: Infecciones respiratorias agudas; Plantas medicinales; Fitoterapia, COVID-19.

\section{Introdução}

Desde a antiguidade, as plantas já eram empregadas com finalidades terapêuticas pelas antigas civilizações da China, Índia, Egito e Grécia, e os saberes repassados a cada geração. Nos dias atuais, essa prática ainda é utilizada no tratamento e/ou cura de enfermidades, sendo considerada além de antiga, também relacionada diretamente com a própria evolução do homem (Alves, 2013).

No Brasil, a utilização das plantas medicinais teve seu primeiro registro no século XVI e o seu uso terapêutico é baseado nas culturas indígenas, africanas e europeias (Argenta, et al., 2011; Braga, 2011). Ademais, o país tem uma rica história com a utilização das plantas medicinais no tratamento dos problemas de saúde da população, uso este construído com base na experiência e transmitido de forma oral (Bruning, et al., 2012).

De acordo com o estudo realizado por Zago (2018), a Índia e o Brasil são os países que nos últimos 22 anos realizaram mais publicações relacionadas a plantas medicinais. Nesse âmbito, tais publicações são necessárias, posto que boa 
parte da população brasileira tem as plantas medicinais como único amparo terapêutico, assim como 2/3 da população mundial. Essas espécies vegetais, cultivadas ou não, são empregadas com fins medicinais, pois possuem em sua composição substâncias capazes de melhorar a saúde humana (Monteiro \& Brandeli, 2017).

Em 11 de março de 2020, a Organização Mundial de Saúde (OMS) definiu a COVID-19 como estado de pandemia (OPAs, 2020). A doença é causada pelo coronavírus da síndrome respiratória aguda grave 2 (SARS-CoV-2), apresentando alto contágio e elevada taxa de mortalidade, principalmente em idosos e pessoas portadoras de comorbidades (Xavier, et al., 2020).

Até o momento, não existe planta, remédio ou vitamina que cure ou previna essa doença (Cavalcanti, 2020). No entanto, muitas pessoas têm procurado alternativas para fortalecerem o sistema imunológico e prevenirem outras patologias, como é o caso das infecções respiratórias agudas.

As infecções respiratórias agudas são as principais doenças que afetam o sistema respiratório, apresentando, na maioria das vezes, etiologia viral. Apesar de serem geralmente consideradas leves, essas infecções podem desencadear quadros mais graves, necessitando de maior atenção e tratamento adequado (Silva Filho, et al., 2017).

As plantas medicinais são bastante utilizadas para o tratamento de doenças respiratórias (Prudente \& Moura, 2013), pois apresentam em sua composição uma série de constituintes capazes de exercer ações anti-inflamatória, antioxidante e antitússica, que são de grande importância no tratamento dessas doenças (Antunes, 2019).

Todavia, vale salientar a importância do conhecimento prévio sobre os riscos e benefícios quanto ao uso da planta medicinal para que esta prática seja benéfica à saúde humana. Desse modo, cabe aos profissionais da saúde a responsabilidade de orientar a população quanto à utilização segura e racional dos fitoterápicos/ plantas medicinais (Badke, et al., 2011).

Nesse contexto, este artigo tem como objetivo relatar a experiência de um projeto de extensão realizado por professores e estudantes dos cursos de Ciências Biológicas e Odontologia da Universidade Federal de Campina Grande, Campus de Patos, Paraíba.

O projeto intitulado "Uso de plantas medicinais pelos professores, estudantes e seus familiares durante a pandemia da COVID-19" visou conscientizar uma comunidade escolar sobre o uso de plantas medicinais utilizadas no controle de infecções respiratórias agudas durante o período pandêmico gerado pela COVID-19.

Esse processo de conscientização se deu através de saberes, ações multiprofissionais, ações de promoção e prevenção, incentivando a participação da sociedade e o cuidado integral com a saúde. Esse estudo também conscientizou os extensionistas que participaram do projeto, bem como, os professores envolvidos nesse processo.

\section{Metodologia}

O projeto foi realizado na Escola Cidadã Integral Técnica Seráfico Nóbrega, no município de São Mamede/PB e teve como público alvo professores, familiares e estudantes do $1^{\circ}$ ao $3^{\circ}$ ano do Ensino Médio. Foi desenvolvido no segundo semestre de 2020, com duração total de 05 meses.

O objetivo do projeto foi repassar informações sobre o uso de plantas medicinais no controle de infecções respiratórias agudas durante a pandemia da COVID-19, esclarecendo ao público pontos essenciais para o uso correto e racional dessas plantas. Salientou-se também que, mesmo essas plantas sendo comumente utilizadas pela população para o tratamento das infecções respiratórias agudas, elas não tratam e nem previnem a COVID-19.

Inicialmente, houve a realização de reuniões síncronas, por meio da plataforma Google Meet®, com os orientadores e extensionistas para o planejamento e elaboração das atividades que seriam desenvolvidas durante o período de vigência do projeto. Posteriormente, realizou-se uma pesquisa com base na literatura sobre as principais plantas com potencial de ação no controle de infecções respiratórias agudas. Foram pesquisados artigos científicos, trabalhos de conclusão de cursos, dissertações e teses, em bases de dados como Google Acadêmico, Scielo e Periódicos CAPES, aplicando na busca a 
combinação das palavras chaves "Plantas Medicinais" e "Infecções Respiratórias Agudas".

Os critérios de inclusão para a seleção dos trabalhos foram: 1) Artigos que envolviam o uso de plantas medicinais para o tratamento ou prevenção dessas doenças; 2) Artigos que abordassem plantas medicinais de uso comum na comunidade; 3 ) Artigos que estivessem no idioma Português. Para critério de exclusão, utilizou-se como parâmetro a não abordagem da temática plantas medicinais e infecções respiratórias agudas.

Ao final da pesquisa, foram selecionados os trabalhos que mais se adequaram aos critérios estabelecidos. Estes estudos reuniam informações sobre as dez plantas medicinais trabalhadas com o público: Acerola (Malpighia emarginata D.C), Amoreira (Morus nigra L.), Alcaçuz (Glycyrrhiza glabra L.), Cumaru (Amburana cearensis (Allemão) A.C.Sm. ), Eucalipto (Eucalyptus globulus Labill), Gengibre (Zingiber officinale Roscoe), Guaco (Mikania glomerata Spreng.), Hortelãda-folha-larga (Plectranthus amboinicus (Lour.) Spreng), Mulungu (Erythrina velutina Willd ) e Romã (Punica granatum L.)

Em seguida, foram elaborados materiais didáticos e informativos, como panfletos, textos e vídeos, sendo postados na plataforma Google Classroom $®$. Também foram realizadas palestras por meio da plataforma Google Meet®, para os estudantes da escola, abordando e discutindo pontos como: manipulação, coleta e uso terapêutico de maneira controlada e correta das plantas medicinais.

Além disso, foi desenvolvido um perfil nas redes sociais (Instagram $\left.{ }^{\circledR}\right)$ sobre a temática do projeto, a fim de garantir um maior alcance das informações e facilitar a comunicação entre os extensionistas e o público. Semanalmente, foram disponibilizados pôsteres contendo curiosidades e informações acerca das plantas medicinais utilizadas no controle de infecções respiratórias agudas, além de vídeos com oficinas ensinando o preparo correto de chás.

\section{Resultados e Discussão}

Devido ao contexto da pandemia da COVID-19, todas as ações realizadas no projeto de extensão ocorreram por meio de plataformas digitais. A plataforma Google Meet ${ }^{\circledR}$ foi utilizada como ferramenta de comunicação com os professores, estudantes e seus familiares que participaram do projeto e na plataforma Google Classroom ${ }^{\circledR}$ foram postados materiais didáticos relacionados ao tema.

É importante salientar que, conforme Moreski, Melo \& Bueno (2018, p. 63), "a Organização Mundial da Saúde reconhece a fitoterapia como uma alternativa de tratamento viável com baixo custo e recomenda o levantamento, identificação, estímulo e orientação do uso das plantas medicinais que possuem eficácia e segurança terapêutica comprovada".

A princípio, as ações executadas no projeto envolveram a realização de palestras abordando aspectos relacionados as infecções respiratórias agudas. Este tipo de infecção acomete o sistema respiratório do indivíduo e se manifesta em um curto espaço de tempo, geralmente sete dias. Podem ocorrer no trato respiratório superior ou inferior, sendo causadas principalmente por vírus ou bactérias (Negrini \& Silva, 2015).

Segundo Gordillo (2018), as doenças respiratórias agudas mais comuns são: bronquite, rinite, sinusite, gripe, resfriado, faringite, amigdalite e pneumonia. As pessoas acometidas com este tipo de infecção podem apresentar sintomas leves, moderados ou intensos, que podem se tornar mais graves e inclusive levar o indivíduo a morte (Lima, et al., 2021).

Por meio de uma revisão de literatura, foram selecionadas dez plantas medicinais capazes de auxiliar no tratamento ou prevenção das infecções respiratórias agudas. Informações como as propriedades terapêuticas e a forma correta de utilização dessas plantas foram apresentadas ao público, bem como possíveis efeitos colaterais ou contraindicações. Também foi ressaltado que, apesar de proporcionarem um alívio dos sintomas causados pelas infecções respiratórias agudas, as plantas apresentadas não são a cura ou a prevenção da COVID-19.

Segundo Melo et al., (2017), várias plantas medicinais podem ser utilizadas para o tratamento de doenças respiratórias, pois possuem em sua composição ampla variedade de compostos químicos, dentre eles: glicosídeo cianogênico, 
mucilagens, taninos e cumarinas, que apresentam diferentes ações farmacológicas como anti-inflamatória, broncodilatadora, expectorante, analgésica e antipirética.

O público-alvo do projeto mostrou-se bastante interativo e a partir dos relatos trazidos percebeu-se que os participantes utilizavam as plantas medicinais de forma frequente. Apesar dos avanços tecnológicos existentes na atualidade, nota-se que a sociedade ainda demonstra grande interesse em utilizar as plantas como recurso terapêutico. Esta escolha é influenciada pelo fato de muitos medicamentos terem preço elevado e causarem irritações no organismo das pessoas que os utilizam de forma constante (Leite, et al., 2015). Em contraste, as plantas medicinais apresentam diversas vantagens como: boa eficácia, baixo custo, poucos efeitos colaterais e o estímulo a hábitos de vida mais saudáveis (Araújo, et al., 2015).

No entanto, Batista et al., (2017) ressaltam que quando utilizadas de forma incorreta, as plantas medicinais podem representar um risco para a saúde, tendo em vista que muitas espécies utilizadas na medicina popular possuem substâncias consideradas tóxicas. Nesse contexto, é imprescindível disseminar o conhecimento científico a respeito da fitoterapia, pois assim a população poderá utilizar esse recurso de forma mais segura e eficaz.

Além das ações realizadas na escola, as informações a respeito das plantas medicinais também foram divulgadas em um perfil do Instagram ${ }^{\circledR}$ (@ conscientizacaoplantasmed). As publicações ocorriam semanalmente por meio de vídeos e textos contendo informações de grande relevância sobre o assunto, como, por exemplo, os benefícios proporcionados pelas plantas medicinais e a forma correta de utilizá-las (Figura 1). Assim, com a utilização das redes sociais, as informações a respeito da temática não ficaram restritas a comunidade beneficiada pelo projeto, mas também atingiram outras parcelas da sociedade.

Figura 1. Publicações no perfil do Instagram contendo informações sobre o uso de plantas medicinais.
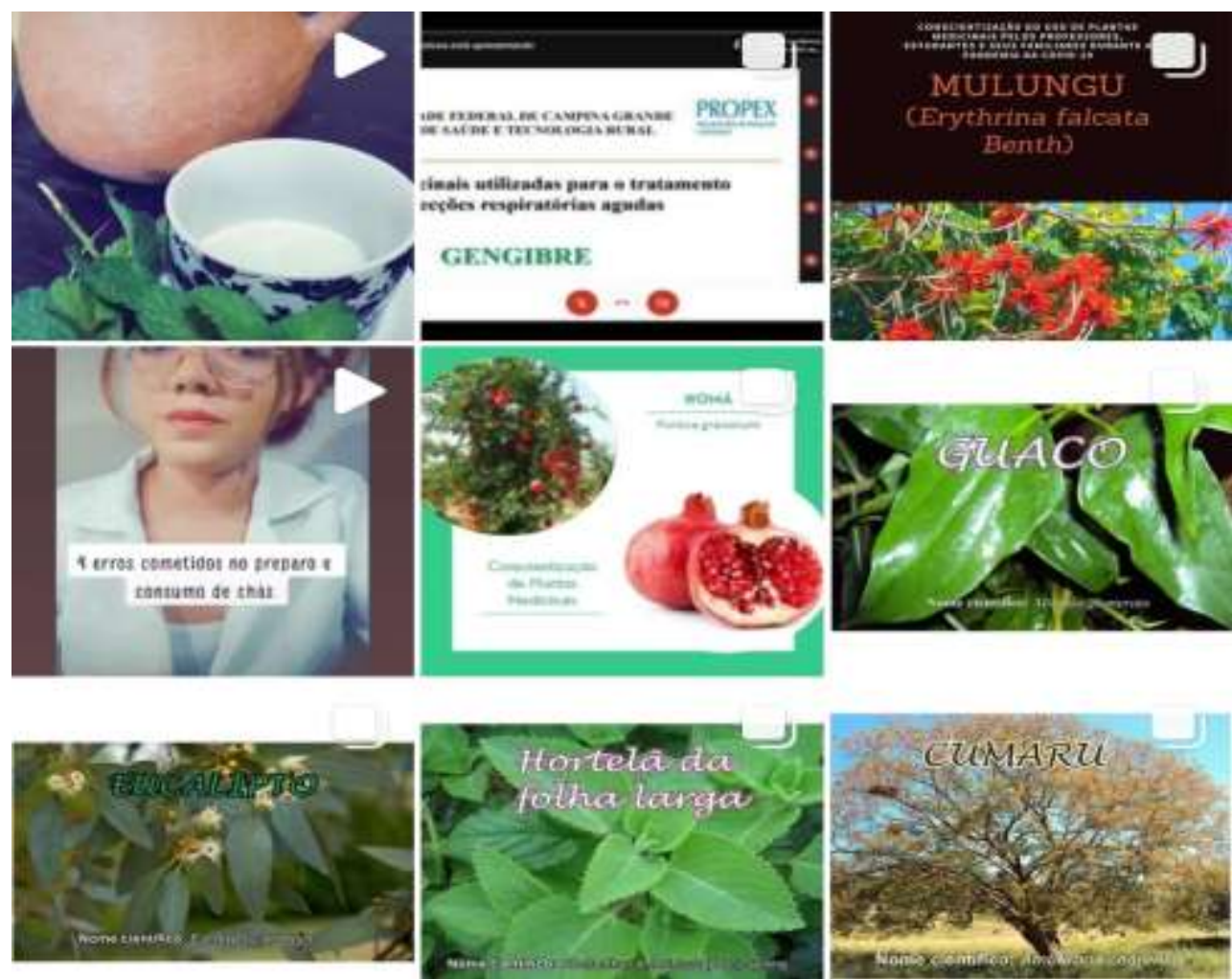

Fonte. Os autores (2021).

Essas informações passadas para a comunidade tiveram como propósito esclarecer os pontos essenciais do uso racional de plantas medicinais no controle de infecções respiratórias agudas durante a pandemia da COVID-19. Para isso, foram escolhidas as principais plantas utilizadas pela população como terapia complementar. 
As plantas medicinais foram escolhidas por serem facilmente cultivadas em hortos caseiros pelos familiares dos alunos. Como é comum uma planta medicinal ser conhecida por diversos nomes, para descrição de cada espécie utilizou-se o nome científico e os nomes populares, facilitando a identificação da planta. Além disso, foi repassado aos participantes do projeto informações para o uso correto dessas plantas, como por exemplo, a parte utilizada, as propriedades medicinais e a forma terapêutica, que variam de acordo com a espécie (Tabela 1).

Tabela 1. Informações repassadas ao público para o uso correto das plantas medicinais trabalhadas no projeto de extensão.

\begin{tabular}{|c|c|c|c|c|}
\hline Nome Científico & Nome popular & Parte utilizada & Propriedades medicinais & Forma terapêutica \\
\hline \multirow[t]{4}{*}{ Malpighia emarginata D.C } & Acerola & Fruto & Antioxidante & In natura \\
\hline & & & Fonte de vitamina A & Suco \\
\hline & & & Fonte de vitaminas do & \\
\hline & & & completo B & \\
\hline \multirow[t]{3}{*}{ Glycyrrhiza glabra $\mathrm{L}$} & Alcaçuz & Raiz & Expectorante & Decocção \\
\hline & & & Antioxidante & \\
\hline & & & Anti-inflamatório & \\
\hline \multirow[t]{3}{*}{ Morus nigra $\mathrm{L}$} & Amoreira & Folhas & Anti-inflamatório & Infusão \\
\hline & & & Antitussígeno & \\
\hline & & & Antioxidantes & \\
\hline Amburana cearenses (Allemão) A. & Cumaru & Sementes & Anti-inflamatória & Infusão \\
\hline \multirow[t]{2}{*}{ C. Sm } & & Folhas & Analgésica & Decocção \\
\hline & & Casca do caule & Bronco-dilatadora & Xarope \\
\hline \multirow[t]{4}{*}{ Eucalyptus globulus Labill } & Eucalipto & Folhas & Antibacteriana & Infusão \\
\hline & & & Expectorante & Inalação de óleos \\
\hline & & & Antisséptico & essenciais \\
\hline & & & Antioxidante & \\
\hline \multirow[t]{4}{*}{ Zingiber officinale Roscoe } & Gengibre & Rizoma & Antioxidante & Decocção \\
\hline & & & Antisséptico & \\
\hline & & & Anti-inflamatório & \\
\hline & & & Expectorante & \\
\hline \multirow[t]{3}{*}{ Mikania glomerata Spreng } & Guaco & Folhas & Bronco-dilatadora & Infusão \\
\hline & & & Expectorante & Xarope \\
\hline & & & Anti-inflamatória & \\
\hline \multirow{3}{*}{$\begin{array}{c}\text { Plectranthus amboinicus (Lour.) } \\
\text { Spreng }\end{array}$} & Hortelã da & Folhas & Anti- inflamatória & Infusão \\
\hline & folha larga & & Antimicrobiano & Xarope \\
\hline & & & Analgésica & \\
\hline \multirow[t]{4}{*}{ Erythrina velutina Willd } & Mulungu & Folhas & Antimicrobiano & Decocção \\
\hline & & & Anti-inflamatório & \\
\hline & & & Analgésico & \\
\hline & & & Ansiolítico & \\
\hline \multirow[t]{5}{*}{ Punica granatun $\mathrm{L}$} & Romã & Folhas & Anti-inflamatória & In natura \\
\hline & & Fruto & Antimicrobiana & Suco \\
\hline & & Casca & Antioxidante & Xarope \\
\hline & & Sementes & & Decocção \\
\hline & & Flores & & \\
\hline
\end{tabular}


Fonte: Os autores, (2021).

Salienta-se que não existem plantas medicinais comprovadas cientificamente para o tratamento da COVID-19. A melhor forma de prevenção ainda é o distanciamento social, higienização das mãos e o uso de máscaras. As plantas medicinais são para utilizar em complementariedade com os tratamentos médicos e como tratamento preventivo para estimular o sistema imunológico.

\section{Conclusão}

Foram selecionadas dez plantas medicinais para serem trabalhadas com o público do projeto de extensão, devido às propriedades terapêuticas que estas apresentam frente as infecções respiratórias agudas. As ações realizadas possibilitaram que os participantes assimilassem conhecimentos em relação à utilização das plantas medicinais, especialmente aquelas capazes de auxiliar no tratamento ou prevenção dessas doenças. Assim, foi possível estabelecer conexão entre o conhecimento popular e o científico permitindo que a comunidade utilizasse a fitoterapia de forma mais consciente e segura.

\section{Referências}

Alves, L. F (2013). Produção de Fitoterápicos no Brasil: História, Problemas e Perspectivas. Revista Virtual de Química, 5 (3), 450-513. doi: 10.5935/19846835.20130038 .

Antunes, A. S. V. (2019). Plantas e Produtos Vegetais com ação no Aparelho Respiratório. 2019. 50f. Dissertação (Mestrado Integrado em Ciências Farmacêuticas). Universidade de Lisboa, 2019.

Araújo, I. F. M., Souza, L. F., Guarçoni, E. A. E., \& Firmo, W. C. A. (2015). O comércio de plantas com propriedades medicinais na cidade de Bacabal, Maranhão, Brasil. Natureza on line, 13 (3), 111-116. http://www.naturezaonline.com.br/natureza/conteudo/pdf/AraujoIFMetal_111-116.pdf.

Argenta, S. C., Argenta, L. C., Giacomelli, S. R., \& Cezarotto, V. S. (2011). Plantas medicinais: cultura popular versus ciência. Vivências, 7 (12), 51-60. https://www.ufpb.br/nephf/contents/documentos/artigos/fitoterapia/plantas-medicinais-cultural-popular-versus-ciencia.pdf.

Badke, M. R. Budó, M. L. D., Silva, F. M., \& Ressel, L. B. (2011). Plantas medicinais: o saber sustentado na prática do cotidiano popular. Revista de Enfermagem, 15 (1), 132-139. doi: 10.1590/S1414-81452011000100019.

Batista, F. T., Lacerda, G. D., Silva., J. R. S., Rodrigues, L. P. T., Feitosa, T. P., \& Mustafa, V. S. (2017). O uso de plantas medicinais na Medicina Veterinária - riscos e benefícios. REVET - Revista Científica do Curso de Medicina Veterinária, 4 (2), 62-74. http://revista.faciplac.edu.br/index.php/Revet/article/view/337.

Braga, C. M. (2011). Histórico da utilização de plantas medicinais. 2011. 24 f. Monografia (Trabalho de Conclusão de Curso) - Consórcio Setentrional de Educação a distância, Universidade de Brasília/ Universidade Estadual do Goiás, Brasília.

Bruning, M. C. R., Mosegui, G. B. G., \& Viana, C. M. M. (2012). A utilização da fitoterapia e de plantas medicinais em unidades básicas de saúde nos municípios de Cascavel e Foz do Iguaçu-Paraná: a visão dos profissionais de saúde. Ciência e Saúde coletiva, 17 (10), 2.675-2.685. doi: 10.1590/S141381232012001000017 .

Cavalcanti, I. M. F. (2020). Plantas medicinais e seus possíveis benefícios no enfrentamento da Covid-19. Belém: RFB Editora.

Gordillo, E. V. (2018). Intervenção Educativa sobre Infecção Respiratória Aguda em crianças de 0 a 12 anos atendidas na UBS Bela Vista no Município de Guairá, Paraná. (2018). 36 f. Monografia (Especialização Multiprofissional na Atenção Básica). Universidade Federal de Santa Catarina, Florianópolis.

Leite, I. A. Moraes, A. M., Do Ó, K. D. S., Carneiro, R. G., \& Leite, C. A. (2015). A Etnobotânica de plantas medicinais no município de São José de Espinharas, Paraíba, Brasil. Biodiversidade, 14 (1), 22-30. https://periodicoscientificos.ufmt.br/ojs/index.php/biodiversidade/article/view/2249.

Lima, R. D. C. S., Xavier, J. M. V., Olinda, R. A., Torquato, S. C., Querino, L. A. L., Baía, S. R. D., Veiga, D. K. E., Araújo, J. M., Costa, W. K. A., \& Figueiredo, R. A. N. (2021). Avaliação da influência do estado nutricional na ocorrência de infecção respiratória aguda em menores de dois anos. Revista Eletrônica Acervo Saúde, 13 (4), 01-09. doi: 10.25248/reas.e6494.2021.

Melo, M. C. S., Vilar, M. S. A., Confessor, M. V. A., \& Vilar, D. A. (2017). Plantas medicinais no tratamento de doenças respiratórias. Anais do II Congresso Brasileiro de Ciências da Saúde. Campina Grande, Realize Editora.

Monteiro, S. C., \& Brandelli, C. L. L. (2017). Farmacobotânica: Aspectos teóricos e aplicação. Porto Alegre: Artmed.

Moreski, D. A. B., Leite-Mello, E. V. S., \& Bueno, F. G. (2018). Ação cicatrizante de plantas medicinais: um estudo de revisão. Arquivos de Ciências da Saúde, 22 (1), 63-69. doi: 10.25110/arqsaude.v22i1.2018.6300.

Negrini, K. L., \& Silva, M. M. O. (2016). Prevenção das infecções respiratórias agudas em crianças de 0-5 anos em uma unidade de saúde da família. Anais do $12^{\circ}$ Congresso Internacional da Rede Unida. 2 (1). 
Research, Society and Development, v. 10, n. 10, e336101019071, 2021

(CC BY 4.0) | ISSN 2525-3409 | DOI: http://dx.doi.org/10.33448/rsd-v10i10.19071

Organização Panamericana de Saúde. (2020) Ongoing Living Update of Potential COVID-19 Therapeutics: summary of rapid systematic reviews. www.paho.org/en/documents/ongoing-living-update-potential-covid-19-therapeutics-summary-rapid-systematic-reviews.

Silva Filho, E. B., Silva, A. L., Santos, A. O., Dall’acqua, D. S. V., \& Souza, L. F. B. (2017). Infecções Respiratórias de Importância Clínica: uma Revisão Sistemática. Revista FIMCA, 4 (1), 07-16.

Xavier, A. R., Silva, J. S., Almeida, J. P. C. L., Conceição, J. F. F., Lacerda, G. S., Kanaan, S. (2020). COVID-19: manifestações clínicas e laboratoriais na infecção pelo novo coronavírus. Jornal Brasileiro de Patologia e Medicina Laboratorial, 56, 01-09. doi: 10.5935/1676-2444.20200049.

Zago, L. M. S. (2018). Vinte e dois anos de pesquisa sobre plantas medicinais: uma análise cienciométrica. Tecnia, 3(1). http://revistas.ifg.edu.br/tecnia/article/view/220/76\#. 